Case Report

\title{
Isolated Cysticercosis of the Abdominal Wall-A Case Report of a Rare Site of Occurrence
}

\author{
Neema Tiwari', Devajit Nath ${ }^{2}$, Savitri Singh ${ }^{3}$, Jyotsna Madan ${ }^{4}$, Sangeeta Tripathi ${ }^{5}$ \\ ${ }^{1}$ Senior Resident, ${ }^{2}$ Assistant Professor, ${ }^{3}$ Associate Professor, ${ }^{4}$ Professor, Dept. of Pathology, Super Specialty Pediatric Hospital \\ and Post Graduate Teaching Institute, Noida, Uttar Pradesh, India. \\ ${ }^{5}$ Professor, Department of Radiology, Super Specialty Pediatric Hospital Post Graduate Teaching Institute, Noida, Uttar Pradesh, \\ India. \\ DOI: https://doi.org/10.24321/2454.8642.201903
}

\section{I $\quad \mathbf{N} \quad \mathbf{F} \quad \mathbf{O}$}

\section{Corresponding Author:}

Devajit Nath, Department of Pathology, Super Specialty Pediatric Hospital Post Graduate Teaching Institute, Noida, Uttar Pradesh, India. E-mail Id:

devajit_nath@yahoo.co.in

Orcid Id:

https://orcid.org/0000-0001-6748-8088

How to cite this article:

Tiwari N, Nath D, Singh S et al. Isolated Cysticercosis of the Abdominal Wall-A Case Report of a Rare Site of Occurrence. Rec Adv Path Lab Med 2019; 5(1): 9-12.

Date of Submission: 2019-04-28

Date of Acceptance: 2019-05-18

\section{$\begin{array}{llllllll}\mathbf{A} & \mathbf{B} & \mathbf{S} & \mathbf{T} & \mathbf{R} & \mathbf{A} & \mathbf{C} & \mathbf{T}\end{array}$}

Pediatric population is the most susceptible age for parasitic infestation and is common in the developing nations where cleanliness and hygiene are an issue and overpopulation and overcrowding are rampantly present. The incidence of cysticercosis is high in India though the exact figures are not known. Human cysticercosis is caused by infestation with larvae of pork tapeworm Taenia solium and is endemic in India. Ingestion of raw or under cooked meat/ pork containing the cysticercosis which spreads through feco-oral route. We present a case of a 6-year- old boy who presented with an upper abdominal wall mass without any other systemic complaints. Fine Needle Aspiration Cytology (FNAC) of the patient was performed and was reported as suspicious for parasitic infestation most likely cysticercosis and was advised excision biopsy. Surgery was performed followed by histopathological examination. A diagnosis of cysticercosis in the histopathology was given. This case highlights the presence of isolated cysticercosis occurring in the abdominal wall which is a rare presentation of the disease.

Keywords: Abdomen, Cysticercosis, Isolated, Rare, Wall

\section{Introduction}

Parasitic infestation is common in the developing nations where cleanliness and hygiene is an issue and overcrowding is rampantly present. Cysticercosis is the infestation of the human body by pork tape worm also called Taenia solium. Overcrowding leads to increased chances of developing a parasitic infestation and cysticercosis cellulosae is one of the common infestations in the human population. ${ }^{1}$ Demographically pediatric age group is the most vulnerable population to be affected. ${ }^{2}$

The incidence of cysticercosis is high in India though the exact figures are not known. Human cysticercosis is caused by infestation with larvae of pork tapeworm Taenia solium through feco-oral route and is endemic in India. ${ }^{3}$ In one study done in Indian population $33.3 \%$ vegetarians developed cysticercosis infection. ${ }^{4}$ The most probable cause for this is ingestion of food and water contaminated by fecal matter containing eggs of cysticercosis, when they have not been washed properly. The life cycle of taenia solium involves man as an intermediate host and pig as a definitive host. ${ }^{5}$ The disease involves many parts of the body, with CNS being the most common organ of involvement. ${ }^{4}$ There are other sites which are infested by this parasite apart from the brain, primarily the soft tissue e.g. muscle etc. Skeletal muscles of extremities are involved and less 
frequently there is involvement of the subcutaneous plane. ${ }^{3}$ Isolated soft tissue cysticercosis without central nervous system involvement is extremely rare. ${ }^{4} \mathrm{~A}$ literature search revealed paucity of data on soft tissue cysticercosis in the pediatric age group. ${ }^{6}$

Patients may be asymptomatic for years, or develop symptoms like nodules cysts or even headache and seizures when CNS is involved. Diagnosis of cysticercosis infestation in the human body can be made by various modalities and the more sensitive and specific ones are, computed tomography (CT), USG, FNAC and histopathology.

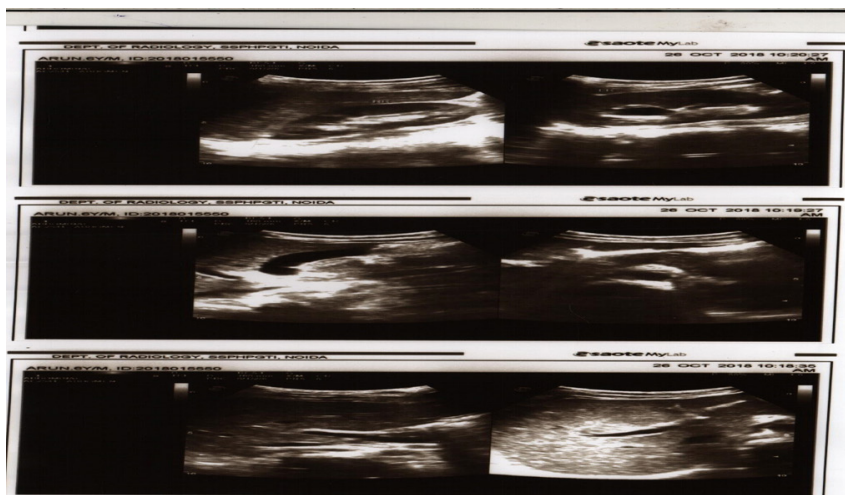

Figure I.A well-defined, avascular, oval cystic lesion with little solid component I I $\times 8 \mathrm{~mm}$ noted in anterior abdominal wall (muscular/ subcutaneous) of let lumbar region s/o cysticercosis

We present a case of a 6-year-old boy who presented with an upper abdominal wall mass which was diagnosed as parasitic infestation suspicious for cysticercosis on FNAC with clinical and radiological correlation, given the paucicellular nature of the aspirate and thereby limiting the final diagnosis on FNAC. Post excision of the lump, histopathology confirmed the diagnosis of cysticercosis.

\section{Case Report}

A 6-year-old boy presented to the Pediatric surgery OPD with complaints of swelling in the upper abdominal wall for approximately one-month duration. The swelling was gradually progressive and painful. No history of trauma to the site of swelling or any other significant past history could be elicited. On examination the swelling was $1.5 \mathrm{x}$ $1.5 \mathrm{cms}$ in the epigastric muscle plain and was firm, mobile and tender.

All the routine investigations were within normal limits however the urine examination showed presence of pus cells and RBCs. Imaging studies (USG) of abdomen wall showed a well-defined partially cystic lesion measuring $11 \times 6 \mathrm{cms}$, with minimal solid component present within the cyst. An impression of infected Cysticercosis was given. A co-existing grade 1 left hydronephrosis with left renal calculi was also reported (Figure 1). An FNAC of the abdominal wall swelling was performed in the Department of Pathology which revealed paucicellular smears with an isolated hooklet like structure and some amorphous eosinophilic membrane like material on a clear background. Correlating clinical findings with FNAC findings, a diagnosis suspicious for parasite? Cysticercosis (Figure 2) was given and an excision biopsy was advised. The lesion was excised and sent for histopathology which revealed presence of eosinophilic sac like wall of the parasite admixed with inflammatory cells thereby conferring a final diagnosis of cysticercosis of the skeletal muscle primarily (Figure 3a, 3b).

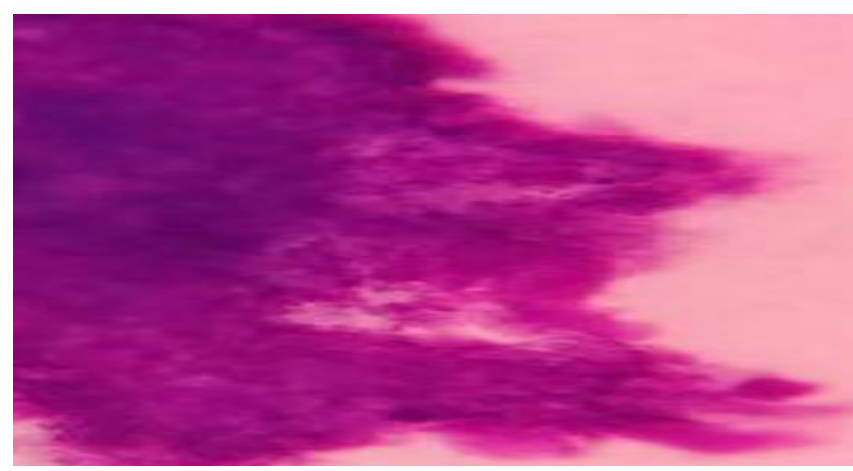

Figure 2.Undulating amorphous membranous material seen on cytology

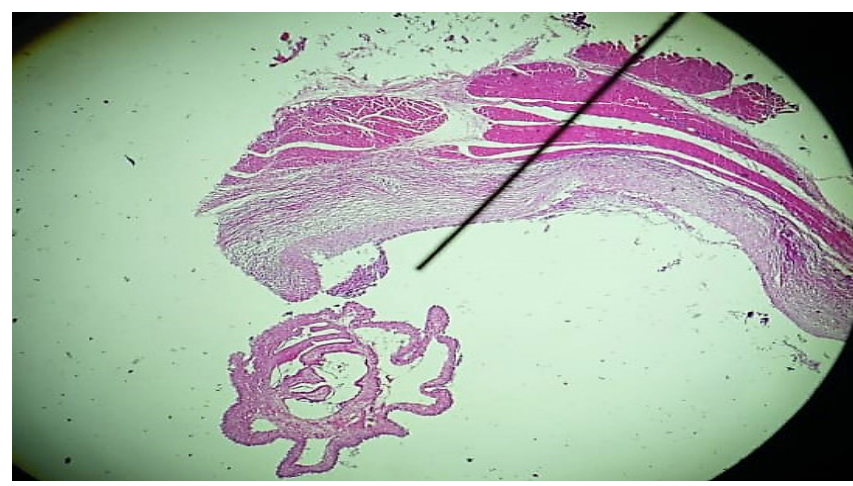

Figure 3(A).Scanner $(10 x)$ view of the cysticercosis wall with surrounding fibrosed tissue

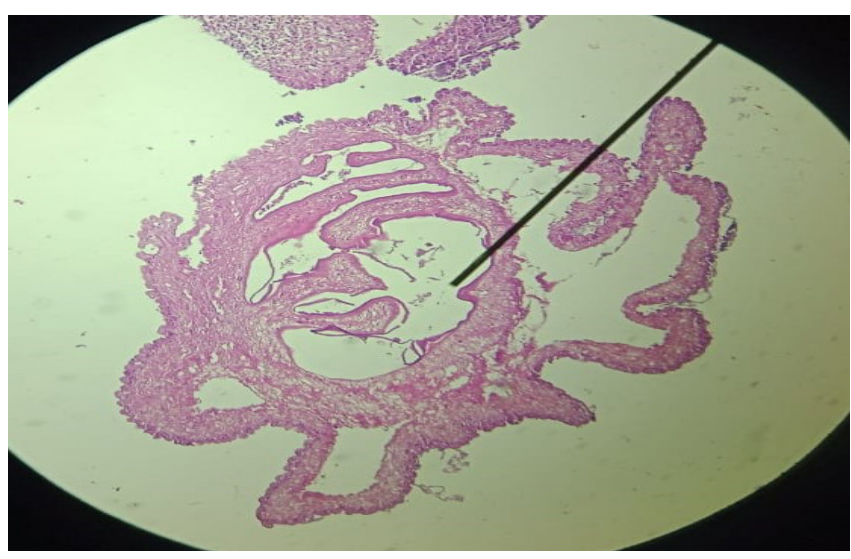

Figure 3(B).High power(40x) view of the cysticercosis parasite showing the layers of the wall 
Patient was started on anthelminthic and discharged. CT/ MRI was not performed as there were no neurological symptoms. Patient has responded well to medication on 6 months follow up.

\section{Discussion}

The systemic manifestation of cysticercosis is caused by dissemination of the larval form of the pork tapeworm, Taenia solium occurs when undercooked pork is commonly consumed or where proper hygiene is not practiced leading to fecal contamination of food and drinks. The incidence of cysticercosis in India is alarmingly high though the exact figures are not known. ${ }^{7}$ In a study done in rural India, the sero-prevalence of cysticercosis was found to be $22.4 \%$ and was known to increase with age. ${ }^{8}$

The normal life cycle of tenia solium involves humans as the definitive hosts and carry intestinal adult tapeworm. Intermittent fecal shedding of proglottids or free eggs occurs, and the intermediate host (normally pigs) ingests the excreted eggs in contaminated food or water. Embryos penetrate the gastrointestinal mucosa of the pig and are haematogenously disseminated to peripheral tissues with formation of larval cysts. When undercooked pork is consumed, an intestinal tapeworm is formed again, completing the life cycle of the worm. ${ }^{9}$

The pathogenesis of human cysticercosis involves reverse peristalsis causing internal regurgitation of the eggs into the stomach when the intestine harbors a gravid worm. ${ }^{7}$ The oncospheres penetrate the intestinal mucosa and develop into cysticercoids after getting carried to various parts of the body including brain, eyes, striated muscles, liver, heart, lungs etc.

The vigorous granulomatous inflammatory reaction that occurs when the larvae die is responsible for all the manifestations of clinical symptoms. ${ }^{10,11}$ Isolated cysticercosis in the subcutaneous plane is a relatively rare form of cysticercosis but should always be kept in the differential diagnosis of subcutaneous swellings. The subcutaneous nodules are usually painless but rupture of the cyst wall causes leakage of fluid and surrounding inflammation with pain in $20 \%$ cases. ${ }^{12}$

Other clinical entities kept in the differential diagnosis depending upon the location of the swelling like a lipoma, ganglion cyst, sebaceous cyst, dermoid, abscess, pyomyositis, tuberculous lymphadenitis, neuroma, sarcoma, myxoma, neurofibroma or fat necrosis. ${ }^{13-15}$

Lesion in the skeletal muscle is asymptomatic but may cause muscular pseudohypertrophy with a heavy parasite burden. Abdominal and chest wall are less commonly involved locations for cysticercosis infestation.

Cysticercosis can disseminate to any part of the body and the diagnosis of cysticercosis involves laboratory investigations coupled with serological tests, imaging studies, Fine Needle Aspiration and tissue diagnosis. Laboratory investigations usually show eosinophilia in the blood and CSF. Serological diagnosis like Indirect hemagglutination test and enzyme linked immunosorbent assay (ELISA) has a sensitivity of about $80 \%$ and can be done in CSF of cases suffering from neurocysticercosis. The enzyme linked immune electro transfer blot (EITB) test is reported to have $100 \%$ sensitivity in patients with two or more viable lesions. ${ }^{18}$

Fine needle aspiration cytology is extremely useful in reaching a diagnosis of soft tissue cysticercosis. The aspirate can be blood stained to clear fluid. Sometimes in approximately $14 \%$ cases pearly white membrane are seen. ${ }^{19}$ The presence of tiny parasitic fragments like spiral larval walls and detached hooklets in an inflammatory back-ground of eosinophils and histiocytes can diagnose cysticercosis with certainty. ${ }^{19}$

Treatment of cysticercosis is high dose anthelminthic therapy with either praziquantel $(50-75 \mathrm{mg} / \mathrm{kg}$ per day for $15-30$ days) or albendazole (10-15 mg/ kg per day for 8 days) ${ }^{19}$

\section{Conclusion}

Isolated cysticercosis is a rare entity and occurrence in the abdominal wall is very uncommon. As in our case the patient is non-pork eating person and occurrence of cysticercosis at a rare site and with non-pork eating habits makes it an interesting case for discussion.

\section{Conflict of Interest: None \\ Reference}

1. Kandukuri MK, Indira V, Soni R et al. Skeletal Muscle Cysticercosis. Int J Healthcare and Biomedical Research 2014; 2(4): 122-126.

2. Gonzalez AE, Lopez Urbina T, Tsang B et al. Transmission dynamics of Tenia Solium and potential for pig to pig transmission. Parasitol Int 2006; 55: 131-135.

3. Kamoji SG, Malipatil PD, Patil MN. Lumpy skin disease: an insight to epilepsy. Journal of Evolution in Medical and Dental Sciences 2015; 4(10): 1723-1726.

4. Husain NA, Stocker TJ, Dehner LP. Pediatric pathology. Wolters Kluwer, Philadelphia 2016.

5. Kumar A, Khan SA, Khan S et al. A study of neurocysticercosis in foothills of Himalaya. Int $J$ Infectious Dis 2006; 10(1): 79-82.

6. Devdhar S, Aggarwal R. Cysticercosis in palpable nodules - a clinico-pathological study with short review of literature. Journal of Medical Science and Research 2013; 4(2): 62.

7. Suchitha S, Vani K, Sunila R et al. Fine needle aspiration cytology of cysticercosis - a case report. Case Rep Infect Dis 2012, Article ID 854704, 2 pages. 
8. Gupta NK, Panchonia A, Jain D. Cysticercosis of breast. Trop Parasitol 2013; 3(2): 148-150.

9. González AE, Lopez Urbina T, Tsang B et al. Transmission dynamics of Taenia solium and potential for pig-to-pig transmission. Parasitol Int 2006; 55: 131-135.

10. Nigam JS, Sharma A. Fine needle aspiration cytology of cysticercosis. J Clin Diagn Res 2013; 7(12): 3123.

11. Elhence P, Bansal R, Sharma $S$ et al. Cysticercosis presenting as cervical lymphadenopathy: a rare presentation in two cases with review of literature. Niger J Clin Pract 2012; 15: 361-363.

12. Kraft R: Cysticercosis: an emerging parasitic disease. Am Fam Physician 2007; 75: 91-98.

13. Naik D, Srinath $M$, Kumar A. Soft tissue cysticercosis - ultrasonographic spectrum of the disease. Indian J Radiol Imaging 2011; 21(1): 60-2.

14. Sinha S, Tiwari A, Sarin YK et al. Isolated soft tissue cysticercosis involving the trunk in children: report of 4 cases. APSP J Case Rep 2013; 4(3): 35.

15. Laxman R, Gupta V, Jawed A. An unusual hand swelling! A report on cysticercosis of intrinsic muscles of the palm. European Orthopedics and Traumatology 2014; 5(2): 165-167.

16. Hawk MW, Shahlaie K, Kim KD et al. Neurocysticercosis: a review. Surg Neurol 2005; 63(2): 123-132.

17. Tamhane TM, Kachewar SG, Lakhkar DL. Imaging in disseminated cysticercosis: a case report and review of literature. Indian J Appl Radiol 2015; 1(1): 105.

18. Gupta S, Gupta S, Mittal A et al. A rare manifestation of cysticercosis infestation. Acta Med Indones 2014; 46(1): 54-57.

19. Agnihotri S, Talwar OP, Pudasaini S et al. Cysticercosis of breast - a case report. Pol J Pathol 2006; 57: 53. 